\title{
Genomic abnormalities of TP53 define distinct risk groups of paediatric B-cell non-Hodgkin lymphoma
}

\author{
Alexander M. Newman ${ }^{1,2,3}$, Masood Zaka ${ }^{1,2,3}$, Peixun Zhou ${ }^{1,2,3}$, Alex E. Blain (iD ${ }^{3}$, Amy Erhorn ${ }^{3}$, Amy Barnard ${ }^{3}$, Rachel E. Crossland ${ }^{3}$, \\ Sarah Wilkinson ${ }^{3}$, Amir Enshaei ${ }^{3}$, Julian De Zordi ${ }^{3}$, Fiona Harding ${ }^{4}$, Mary Taj ${ }^{5}$, Katrina M. Wood ${ }^{6}$, Despina Televantou ${ }^{6}$, \\ Suzanne D. Turner ${ }^{7,8}{ }^{7,8}$, G. A. Amos Burke $\mathbb{I D}^{9}$, Christine J. Harrison ${ }^{3}$, Simon Bomken ${ }^{3,10}$, Chris M. Bacon ${ }^{3,6}$ and Vikki Rand (iD ${ }^{1,2,3 凶}$
}

(c) The Author(s) 2021

Children with B-cell non-Hodgkin lymphoma (B-NHL) have an excellent chance of survival, however, current clinical risk stratification places as many as half of patients in a high-risk group receiving very intensive chemo-immunotherapy. TP53 alterations are associated with adverse outcome in many malignancies; however, whilst common in paediatric B-NHL, their utility as a risk classifier is unknown. We evaluated the clinical significance of TP53 abnormalities (mutations, deletion and/or copy number neutral loss of heterozygosity) in a large UK paediatric B-NHL cohort and determined their impact on survival. TP53 abnormalities were present in $54.7 \%$ of cases and were independently associated with a significantly inferior survival compared to those without a TP53 abnormality (PFS $70.0 \%$ vs $100 \%, p<0.001$, OS $78.0 \%$ vs $100 \%, p=0.002$ ). Moreover, amongst patients clinically defined as high-risk (stage III with high LDH or stage IV), those without a TP53 abnormality have superior survival compared to those with TP53 abnormalities (PFS $100 \%$ vs $55.6 \%, p=0.005$, OS $100 \%$ vs $66.7 \%, p=0.019$ ). Biallelic TP53 abnormalities were either maintained from the presentation or acquired at progression in all paired diagnosis/progression Burkitt lymphoma cases. TP53 abnormalities thus define clinical risk groups within paediatric B-NHL and offer a novel molecular risk stratifier, allowing more personalised treatment protocols.

Leukemia (2022) 36:781-789; https://doi.org/10.1038/s41375-021-01444-6

\section{INTRODUCTION}

Current treatments for high-grade paediatric B-cell non-Hodgkin lymphoma (B-NHL) in resource-rich countries are extremely effective, with over $93 \%$ of children being cured [1-5]. Following the recent demonstration of the benefit of the anti-CD20 monoclonal antibody rituximab, these same rates of cure are now achieved even in patients presenting with established highrisk clinical features (high lactate dehydrogenase (LDH), bone marrow (BM) and/or central nervous system (CNS) disease) [5]. This success, however, has required the use of intensive multiagent chemo-immunotherapy regimens, associated with significant, predominantly acute, toxicity such as infection and mucositis as well as a small risk of long-term neurological side effects and second malignancy [6-8]. Accordingly, reduction in treatment intensity for at least some patients is a key objective. Unfortunately, previous attempts to reduce treatment intensity in highrisk bone marrow/CNS-positive patients resulted in unacceptable deterioration in survival, highlighting the need for biomarkerdriven risk stratification to complement currently established clinical and laboratory risk features $[2,9]$.

In contrast to the excellent overall survival currently achieved, the outcome for children with primary refractory or relapsed $\mathrm{B}-\mathrm{NHL}$ is extremely poor, with fewer than $30 \%$ successfully salvaged despite the routine use of high-dose chemotherapy and stem cell rescue [10-13]. Mounting toxicity in this heavily pretreated group means that escalating intensity alone cannot provide the solution to improving outcome in these patients [14]. Instead, a more comprehensive understanding of the biological drivers of therapy resistance is essential to support the development of more effective and less toxic targeted therapies for this group of patients [15].

Genomic studies have greatly improved our understanding of the pathogenesis and clinically relevant heterogeneity of both Burkitt lymphoma (BL) and diffuse large B cell lymphoma (DLBCL)

\footnotetext{
${ }^{1}$ School of Health \& Life Sciences, Teesside University, Middlesbrough, UK. ${ }^{2}$ National Horizons Centre, Teesside University, 38 John Dixon Lane, Darlington, UK. ${ }^{3}$ Wolfson Childhood Cancer Research Centre, Translational and Clinical Research Institute, Newcastle University, Newcastle upon Tyne, UK. ${ }^{4}$ Newcastle Genetics Laboratory, Newcastle upon Tyne Hospitals NHS Foundation Trust, Newcastle upon Tyne, UK. ${ }^{5}$ Department of Paediatric Oncology, Royal Marsden Hospital, Sutton, Surrey, UK. ${ }^{6}$ Department of Cellular Pathology, Newcastle upon Tyne Hospitals NHS Foundation Trust, Newcastle upon Tyne, UK. ${ }^{7}$ Division of Cellular and Molecular Pathology, Department of Pathology, University of Cambridge, Addenbrooke's Hospital, Cambridge, UK. ${ }^{8}$ CEITEC, Masaryk University, Brno, Czech Republic. ${ }^{9}$ Department of Paediatric Haematology, Oncology and Palliative Care, Addenbrooke's Hospital, Cambridge University Hospitals NHS Foundation Trust, Cambridge, UK. ${ }^{10}$ Department of Paediatric and Adolescent Haematology and Oncology, The Great North Children's Hospital, Newcastle upon Tyne Hospitals NHS Foundation Trust, Newcastle upon Tyne, UK. ${ }^{凶}$ email: v.rand@tees.ac.uk
}

Received: 2 March 2021 Revised: 26 September 2021 Accepted: 29 September 2021

Published online: 21 October 2021 
[16-28]. Amongst their findings is the frequent mutation in both diseases of the tumour suppressor gene TP53, inactivation of which has an established role in lymphomagenesis [29-33]. TP53 mutation, often accompanied by loss of heterozygosity, is present in $25-50 \%$ of sporadic BLs and approximately $20 \%$ of adult DLBCLs at diagnosis, while a recent report suggested a lower incidence amongst paediatric large B-cell lymphomas [16-22, 24-28, 34-36]. Consistently, TP53 mutations have been shown to be associated with adverse clinical outcomes in adult aggressive B-cell lymphoma, including DLBCL, and other lymphoid neoplasms [24, 34-38]. In chronic lymphocytic leukaemia (CLL), they are associated with a poor response to chemotherapy and detection of TP53 mutation and $17 p$ deletion is used to guide therapeutic decisions [39]. However, to date, no study has incorporated analysis of clinical outcome with a detailed characterisation of TP53 alterations in paediatric B-NHL where BL predominates, and the potential utility of TP53 status as a clinical risk stratifier in this age group has remained unclear.

To address this question, we collected and analysed a clinically annotated cohort of 95 paediatric B-NHL patients from the UK. Using sequencing and copy number (CN) microarray data we show that TP53 abnormalities at presentation are associated with disease progression and poor outcome. Importantly, the absence of TP53 abnormalities is associated with an extremely low risk of relapse even in high-risk patients defined by high tumour stage and LDH. Moreover, we demonstrate that biallelic TP53 abnormalities are either maintained or acquired at the time of disease progression, implicating loss of TP53 function in the development of treatment resistance. Finally, we show that TP53 abnormalities are associated with complex chromosomal copy number profiles, identifying a potential mechanism underlying the evolution of the chemo-resistant disease.

\section{METHODS \\ Patients and clinical samples}

B-NHL samples from UK hospitals registered with the Children's Cancer and Leukaemia Group (CCLG) Tissue Bank between 1993 and 2014 were obtained following informed consent from participants or their parent/ guardian. A minimum of three-years follow-up was obtained for all survivors. Lymphomas were re-classified according to the World Health Organisation (WHO) Classification of Tumours of Haematopoietic and Lymphoid Tissues [40]. IG-MYC status was confirmed by fluorescence in situ hybridisation (FISH) as described in Supplementary methods. The cohort comprised BL $(n=64)$, DLBCL $(n=19)$, Burkitt-like lymphoma with 11q aberration (BLL-11q, $n=5$ ) and remaining cases which could not be fully classified (B-NHL, NOS $n=7$ ).

\section{TP53 mutation and 17p copy number analysis}

TP53 mutational status was assessed using whole-exome sequencing (WES, $n=90)$ or Sanger sequencing of exons 5 to $8(n=5)$. WES data were generated using Illumina Nextera Exome enrichment $(n=89)$ or TWIST Human Core Exome kit $(n=1)$ and sequenced on an Illumina NovaSeq within the Newcastle University Genomics Core Facility or Illumina HiSeq by Eurofins Genomics (Germany). Data were analysed using the Genome Analysis Toolkit (GATK 3.7) and variants called using Mutect2. PCR products for Sanger sequencing were amplified using primers designed for TP53 (Supplementary Table 1) and sequenced by Eurofins Genomics. WES base calls were confirmed by Sanger sequencing in 39 cases, with $100 \%$ concordance between sequencing methods.

Copy number alterations (CNAs) of $17 p$ and other chromosomes were identified using Affymetrix Cytoscan HD, Genome-wide Human SNP Array 6.0 or OncoScan arrays performed by Eurofins Genomics. Raw data were analysed and visualised in Nexus Copy Number 10.0 (BioDiscovery) to detect CNAs and copy number neutral loss of heterozygosity (CNN-LOH) in all samples.

Chromosomal complexity analysis. Complex patterns of chromosomal copy number abnormality were defined as those with a fluctuation between two or more copy number states involving two or more individual segments $\geq 100 \mathrm{~kb}$ on a chromosome arm. These included chromothripsis-like patterns of alternation between two copy number states as well as stepwise increase in copy number of chromosome arms. This definition excludes regions with heterozygous deletion followed by homozygous deletion, single regions of gain, deletion or CNN-LOH, and abnormalities affecting alternate chromosome arms.

Statistical analyses. Estimates of overall survival (OS) and progression-free survival (PFS) were calculated and compared using Kaplan-Meier methods, log-rank tests and Cox-regression models. OS was defined as the time from diagnosis to death from any cause, with censoring at the date of the last contact. PFS was defined as the date of diagnosis to the time of disease progression or death. We report three-year OS and PFS survival rates. All variables conformed to the proportional hazards assumption. Other comparisons were performed using Fisher's exact test. Analyses were performed using $\mathrm{R}$ Bioconductor packages 'survival' for univariate and multivariate analysis and 'survminer' for visualisation of Kaplan-Meier survival curves.

\section{RESULTS}

\section{Patient demographics and clinical characteristics}

The cohort consisted of the diagnostic tumour samples from 95 UK paediatric B-NHL patients. In total, 89 of the 95 (94\%) cases were uniformly treated on $\mathrm{FAB} / \mathrm{LMB} 96$ protocols (trial or interim guidelines) and had a complete follow-up with a median followup of 66.2 months (1-270.4 months) (Table 1). In keeping with previously published clinical trial cohorts [4, 41], the 89 FAB/ LMB96-treated cases in the present study demonstrated a median age of 8 years, a male predominance of 3.4:1, a predominance (67\%) of Burkitt lymphoma, a majority of high stage (III/IV) patients (73\%), bone marrow disease in $18 \%$ and CNS disease in $6 \%$ of cases. MYC status was available for $84 / 89$ samples (five cases failed FISH or had no available material), amongst which $63(75 \%)$ had an IG-MYC translocation, including 56/58 BL, 4/16 DLBCL, 3/5 B$\mathrm{NHL}, \mathrm{NOS}$ and $0 / 5$ BLL-11q cases. Survival estimates at three years for PFS and OS were $83.1 \%(95 \% \mathrm{Cl} 75.7-91.3)$ and $87.6 \%(95 \% \mathrm{Cl}$ 81.1-94.8), respectively. For those patients with disease progression (primary refractory or relapsed disease), the median time from the initial diagnosis to progression was 4.5 months (range 2.8-7.7 months). The additional 6 of the 95 cases (4 BL, $1 \mathrm{DLBCL}$ and $1 \mathrm{~B}-\mathrm{NHL}, \mathrm{NOS}$ ) were included in the genomic analysis only.

\section{Genomic analysis of the TP53 locus}

TP53 mutations were found in 46/95 (48.4\%) cases: 37 cases had a single non-synonymous somatic mutation, eight had two different somatic mutations and one had a germline mutation (DLBCL with LiFraumeni syndrome) (Fig. 1A, Supplementary Table 2). TP53 mutations were found in 37/64 (57.8\%) BL, 4/19 (21.0\%) DLBCL, 4/7 (57.1\%) B$\mathrm{NHL}$, NOS cases and 1/5 (20.0\%) BLL-11q cases. All three DLBCL cases with somatic TP53 mutations and all four mutated B-NHL, NOS cases carried IG-MYC rearrangements. The 35 distinct mutations included 33 missense mutations and two deletions leading to frameshifts. All but two mutations involved the DNA binding domain and experimental data from the UMD and IARC TP53 databases showed all but two of the mutations to be functionally deleterious $[42,43]$. As seen in other cancers $[42,44]$, the most commonly mutated residues were R175 ( $n$ $=6), \mathrm{G} 245(n=5), \mathrm{R} 248(n=7)$ and R273 $(n=4)$.

Next, we analysed $17 p$ copy number alteration and identified deletions in 15/95 (15.8\%) cases: 10/64 (15.6\%) BL, 4/19 (21.0\%) DLBCL and 1/7 (14.3\%) B-NHL, NOS. The median region of deletion was $20.6 \mathrm{Mb}$ (range $17.9-22.2 \mathrm{Mb}$ ), resulting in deletion of $>80 \%$ of 17p (Fig. 1B). Additionally, 8/95 cases (8.4\%) showed CNN-LOH: 7/ 64 (10.9\%) BL and 1/19 (5.3\%) DLBCL. The median region of CNN$\mathrm{LOH}$ was $19.5 \mathrm{Mb}$ (range 12.9-22.3 Mb), again covering $>80 \%$ of $17 p$ (Fig. 1C). All 17p deletions and CNN-LOH involved the TP53 locus. The presence or absence of TP53 copy number deletion was assessed by FISH in 76/95 cases: 49/64 BL, 16/19 DLBCL, 5/5 BLL$11 \mathrm{q}$ and 6/7 B-NHL, NOS (Supplementary Table 2). These included 12/15 cases with TP53 deletion, 5/8 with CNN-LOH and 59/72 with neither. In each case, the FISH results were concordant with the copy number array findings. 
Table 1. Clinical and cytogenetic characteristics of the FAB/LMB96treated paediatric B-NHL cases.

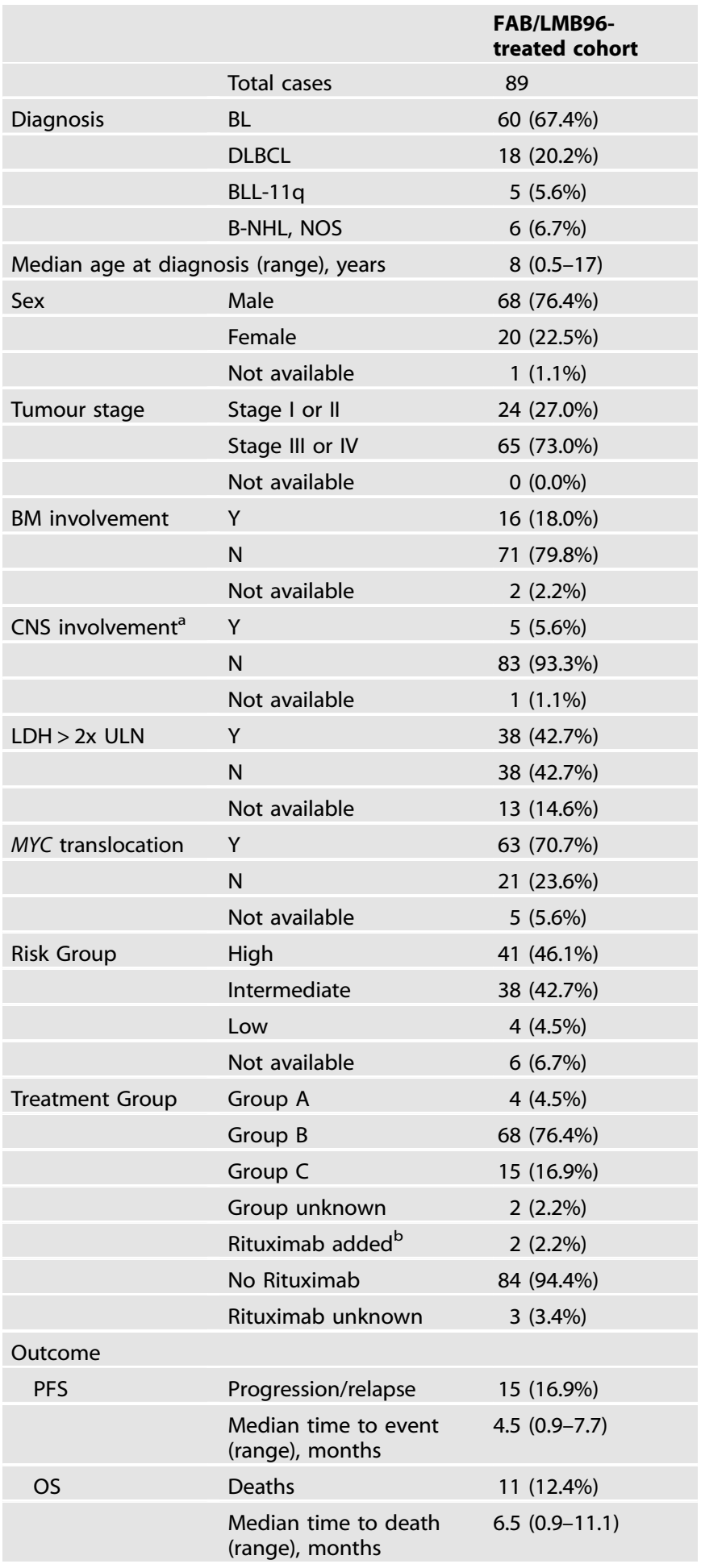

BL Burkitt lymphoma, DLBCL diffuse large B-cell lymphoma, BLL-11q Burkittlike lymphoma with $11 \mathrm{q}$ aberrations, B-NHL, NOS B-cell non-Hodgkin lymphoma not otherwise specified, $Y$ Yes, $N$ No, "-" no event, hazard ratio not reported, $B M$ bone marrow, CNS central nervous system, CSF cerebrospinal fluid, $L D H$ lactate dehydrogenase, ULN upper limit of normal, PFS progression-free survival, OS overall survival.

a One case had CSF involvement.

${ }^{\mathrm{b}}$ Two cases received rituximab from the start of first-line therapy, two other patients received rituximab following switch to group $C$ therapy following post-CYM-1 reassessment.
Combining the mutation and CN data for the TP53 locus showed that abnormalities are common at presentation with 52/ 95 (54.7\%) cases harbouring at least one abnormality and functionally inactivating biallelic events present in 26/95 (27.4\%) cases (Fig. 1D; Supplementary Table 2). Of the 26 cases with monoallelic TP53 abnormalities, 20 had a single somatic heterozygous mutation (median variant allele frequency 39\%, range $18-50 \%)$, four had a deletion and two had CNN-LOH. Among the 26 cases with biallelic abnormalities, 17 had TP53 mutation together with a deletion or CNN-LOH, two had a homozygous mutation and seven had compound heterozygous mutations. The correlation between p53 protein expression and TP53 status was assessed by $\mathrm{p} 53$ immunohistochemistry (IHC) in a subset of cases. In most cases, TP53 mutation was associated with overexpression of p53 protein but the correlation between p53 expression levels and TP53 status was variable and IHC could not reliably detect the range of genomic alterations (Supplementary Table 3).

\section{TP53 abnormalities are associated with chromosomal complexity}

Genomic TP53 alterations have been associated with genomic complexity in DLBCL and other tumours [21, 33, 44-46]. In this study, TP53 abnormalities were not associated with the number of CNAs or the percentage of genome altered (Supplementary Table 4). However, TP53 abnormalities were associated with complex CN profiles of specific chromosomes. (Fig. 2; Supplementary Table 5). Excluding BLL-11q, which is defined by a complex 11q rearrangement, chromosomes with complex CNAs were present in 30/51 cases with TP53 abnormality but only in 5/ 39 cases with wild-type TP53 $(p<0.001)$. This association was particularly strong for $\mathrm{BL}$, in which $24 / 40(60 \%)$ TP53 abnormal cases harboured a complex chromosome compared to only $1 / 24$ (4.2\%) TP53 wild-type cases $(p=0.001)$. The Li-Fraumeni syndrome case (DLBCL with TP53 biallelic abnormality) demonstrated the highest number of complex CNAs within the whole cohort, but otherwise there was no association between TP53 abnormalities and complex CNAs in DLBCL cases.

Most notably, complex CNAs of $1 \mathrm{q}, 11 \mathrm{q}$ and $13 \mathrm{q}$ were associated with biallelic TP53 abnormalities $(p<0.05)$ (Fig. 2). Complexity of $1 \mathrm{q}$ typically involved stepwise increases in copy number, with only two cases having telomeric deletion (Supplementary Fig. 1). Both chromosome arms $11 q$ and $13 q$ recurrently showed a pattern of a region of gain adjacent to telomeric deletion, similar to the profile defining BLL-11q cases [47] (Supplementary Figs. 2-3). All ten cases with this characteristic gain-telomeric deletion pattern of $13 q$ (here termed $13 q^{\text {plex }}$ ) were BL (Supplementary Table 5). The MIR17HG locus mapped within the region of $13 q \mathrm{CN}$ gain/amplification, notably located in the highest peak for $9 / 10(90 \%) 13 q^{\text {plex }}$ cases (Supplementary Fig. 3).

Survival analysis of TP53 abnormalities in paediatric B-NHL The clinical characteristics of cases with or without a TP53 abnormality are presented in Supplementary Table 6. Univariate survival analyses identified a significantly adverse PFS for patients with high disease stage, bone marrow involvement or high LDH (Supplementary Table 7). CNS involvement was associated with a hazard ratio of 3.0 but did not reach significance, likely due to the low number of cases $(n=5)$. Those with mutations had significantly inferior three-year survival compared to those without (PFS $66.7 \%$ (95\% Cl 54.2-82.0) vs $100 \%$ (95\% Cl 100-100), $p<0.001$ and OS $75.6 \%(95 \% \mathrm{Cl} 64.0-89.2)$ vs $100 \%(95 \% \mathrm{Cl} 100-100), p<0.001)$ (Supplementary Fig. 4A, B), although no hazard ratio could be reported due to the absence of events in cases without TP53 mutation. Likewise, 17p CNN-LOH involving TP53 was also associated with worse outcome (PFS HR 4.9 (95\% Cl 1.6-15.6), $p=0.007$; OS HR 4.7 (95\% Cl 1.2-17.7), $p=0.023$ ) (Supplementary Fig. 4C, D, Supplementary Table 7). In contrast, as previously reported in the context of the FAB/LMB96 trial, univariate analysis 


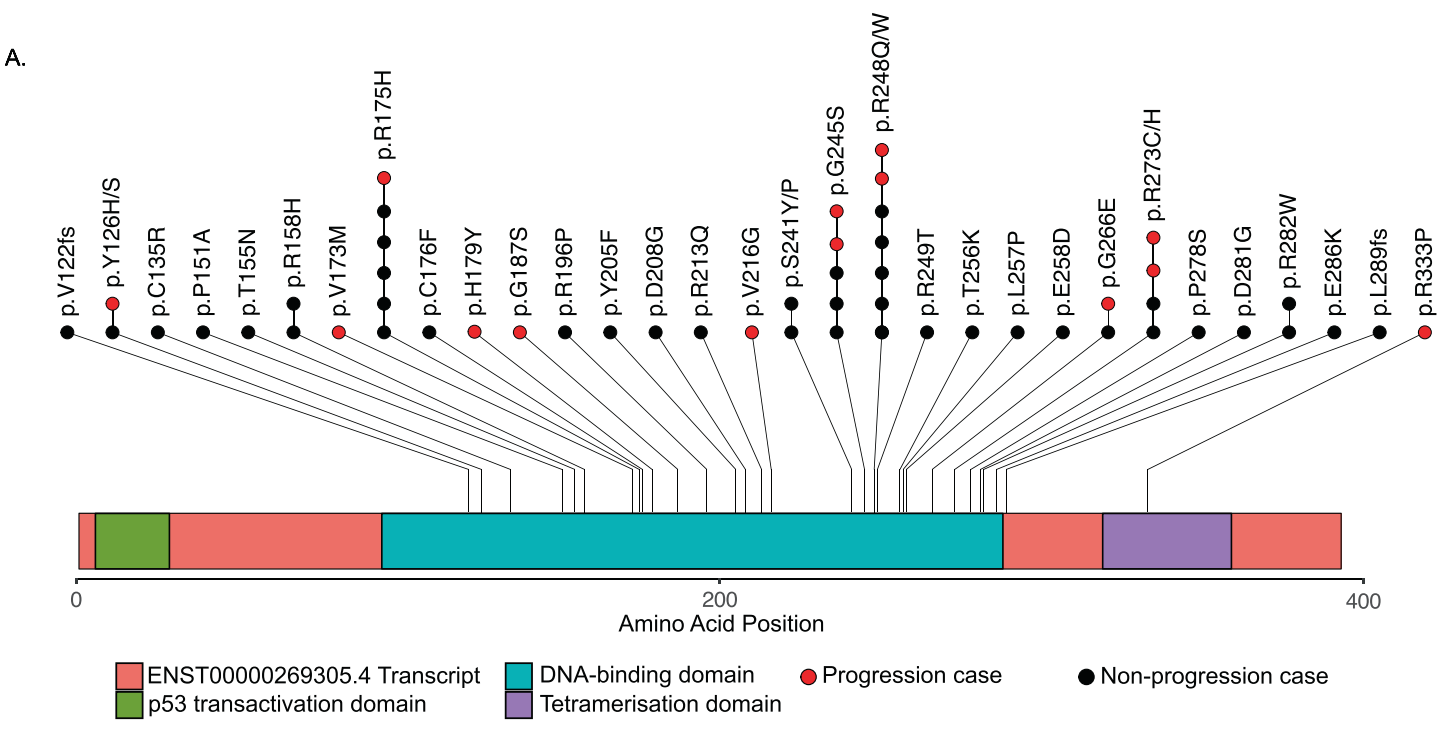

B.

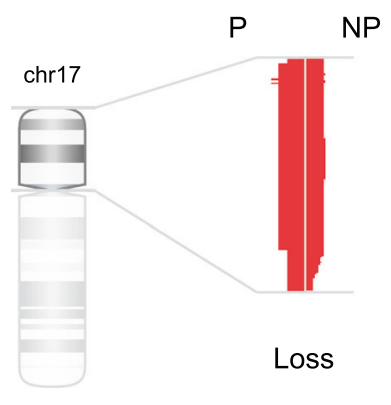

C.

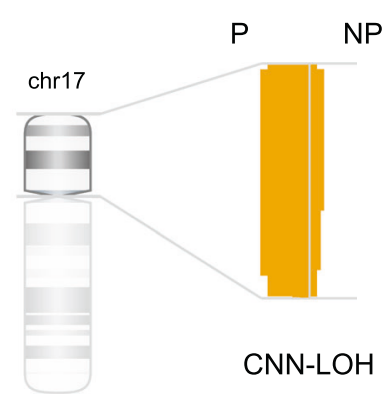

D.

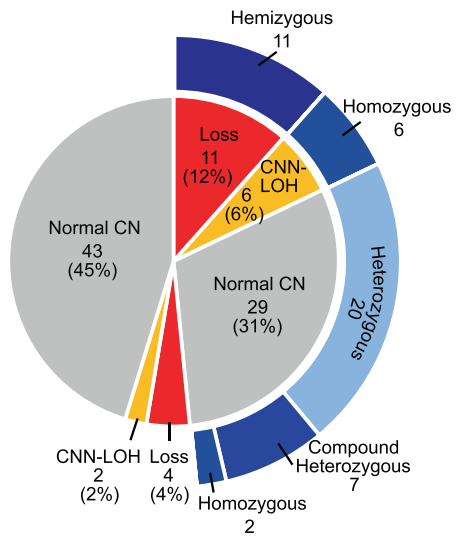

Fig. 1 TP53 mutations and genomic copy number abnormalities are detected in a high proportion of paediatric B-NHL cases. (A) A lolliplot showing 53 somatic and one germline mutation (R248Q detected in a Li-Fraumeni syndrome case). (B) Deletion of the TP53 locus was detected in 3/14 patients with disease progression (P) and 12/78 with no disease progression (NP). (C) CNN-LOH of the TP53 locus was detected in 5/14 patients with disease progression (P) and 3/78 with no disease progression (NP). (D) Representation of co-occurrence of TP53 abnormalities. Inner circle represents TP53 copy number status; outer ring represents TP53 mutation status.

showed that, agnostic of TP53 mutation status, cases with deletion of $17 p$ including the TP53 locus did not have inferior outcome (Supplementary Fig. 4E, F, Supplementary Table 7) [2].

Combining the TP53 locus alterations (Fig. 3A), patients with at least one TP53 abnormality had a significantly inferior 3-year survival compared to those with no abnormality (PFS 70.0\% $(95 \%$ $\mathrm{Cl} 58.4-83.9)$ vs $100(95 \% \mathrm{Cl} 100-100), p<0.001$ and OS $78.0 \%$ (95\% Cl 67.3-90.4) vs 100 (95\% Cl 100-100), $p=0.002$ ) (Fig. 3B, C). Importantly, those patients without any TP53 abnormality at initial diagnosis had a PFS of $100 \%(95 \% \mathrm{Cl} 100-100)$ and an OS of $100 \%$ (95\% Cl 100-100). Most TP53 abnormalities were seen in patients diagnosed with BL and the presence of any TP53 abnormality remained adversely prognostic within this subgroup (PFS 67.5\% (95\% Cl 54.4-83.7) vs $100 \%(95 \% \mathrm{Cl} 100-100), p=0.005$ and OS: $75 \%(95 \% \mathrm{Cl}$ 62.7-89.7) vs $100(95 \% \mathrm{Cl} 100-100, p=0.017)$ (Supplementary Fig. 5). Both monoallelic and biallelic TP53 abnormalities were associated with adverse outcome, when compared with TP53 wild-type cases (monoallelic PFS 76.0\% (95\% Cl 61.0-94.7) vs $100 \%(95 \% \mathrm{Cl} 100-100), p=0.001$ and OS $80.0 \%(95 \% \mathrm{Cl} 65.8-97.3)$ vs $100 \%(95 \% \mathrm{Cl} 100-100), p=0.004 ;$ biallelic PFS $64.0 \%$ (95\% Cl 47.7-85.9) vs $100 \%$ (95\% Cl 100-100), $p<0.001$ and OS $76.0 \%(95 \% \mathrm{Cl} 61.0-94.7)$ vs $100(95 \% \mathrm{Cl}$ 100-100) $p=0.001$ ), but with no significant difference identified between these two groups (Fig. 3D, E). There was no association between prognosis and either complex CNA patterns of 1q, 11q or 13q (Supplementary Table 8) or gain of MIR17HG. Neither was there a difference in outcome for patients with or without any complex CNA (PFS HR 1.7 (95\% Cl 0.6-4.7), $p=0.295$; OS HR 1.8 (95\% Cl 0.5-5.9), $p=0.332$ ).

Multivariate analysis was performed to assess the impact of TP53 abnormalities alongside established high-risk clinical factors (BM and CNS involvement, stage and high LDH) (Supplementary Table 7). Due to the lack of an event amongst the TP53 wild-type patients included in this analysis, we are unable to report a hazard ratio for TP53 status; however, having included this strong prognostic factor in the model, those established high-risk clinical factors were not independently significant.

TP53 status risk stratifies patients with clinically defined highrisk disease

The Inter-B-NHL ritux 2010 trial (NCT01516580) has recently demonstrated the survival benefit of adding rituximab to modified FAB/LMB96 chemotherapy for patients with high-risk disease (stage III and LDH > 2x ULN, stage IV or Burkitt leukaemia) [5]. Nevertheless, rituximab-chemotherapy was associated with increased incidence of prolonged hypogammaglobulinaemia 


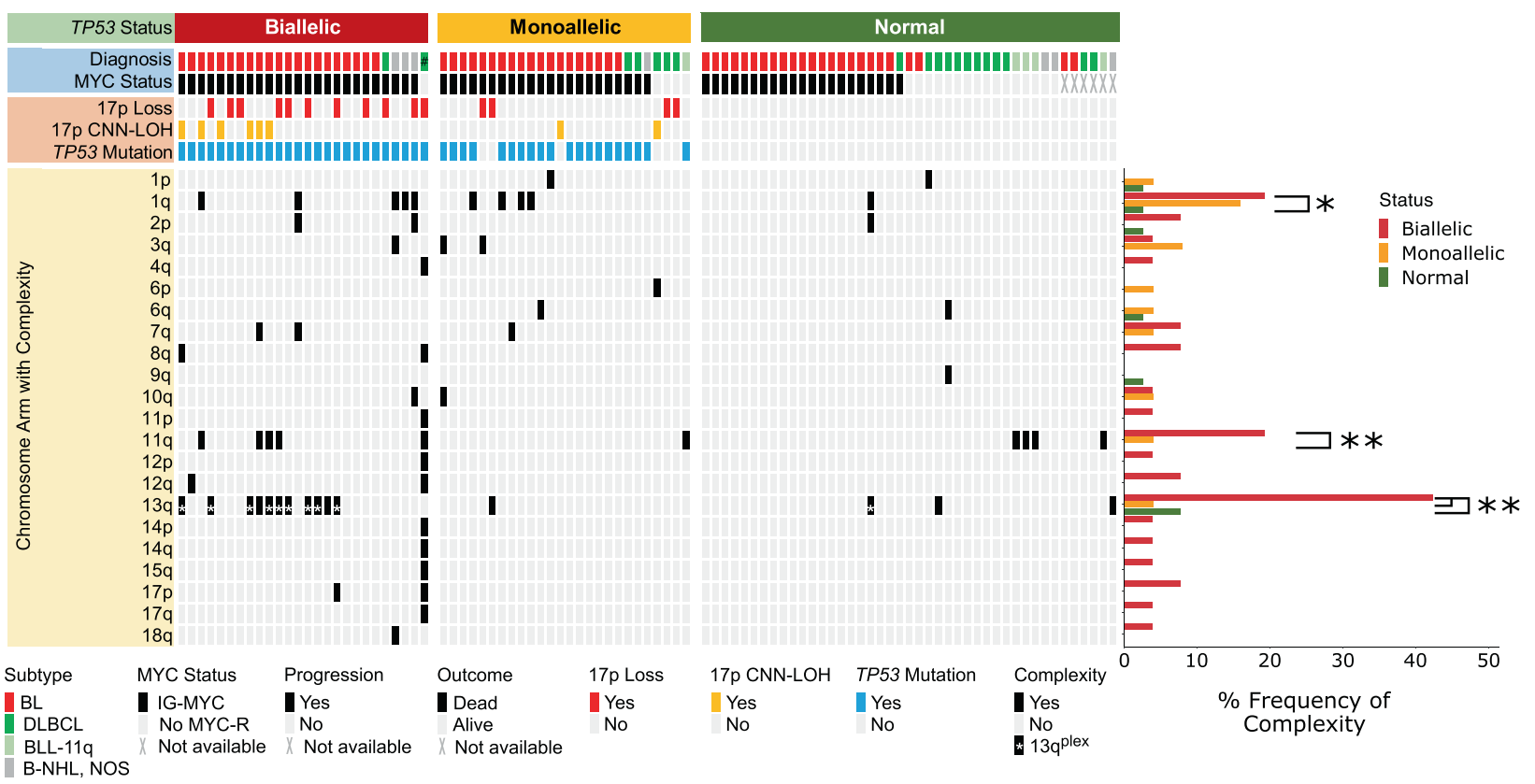

Fig. 2 A pattern of complex chromosomal abnormalities in paediatric B-NHL is associated with TP53 status. Oncoplot showing TP53 status (upper panel) and associated complex chromosomes (lower panel). Histogram displaying the frequency of complex abnormalities of each chromosome arm in biallelic, monoallelic and TP53 normal groups. As a diagnosis of BLL-11q is determined by the presence of a complex 11q rearrangement, these five cases were excluded from this analysis. TP53 Biallelic with 1q complexity vs TP53 Normal with 1q complexity, $p=0.026$; TP53 Biallelic with 11q complexity vs TP53 Normal with 11q complexity, $p=0.006$. TP53 Biallelic with $13 q$ complexity vs TP53 Normal with $13 q$ complexity, $p=0.001$; TP53 Biallelic with $13 q$ complexity vs TP53 Monoallelic with $13 q$ complexity, $p=0.002$. Fisher's Exact test ${ }^{*}=p<0.05$, ${ }^{* *}=p$ $<0.01 ;{ }^{\#}$ Li-Fraumeni syndrome case.

A.

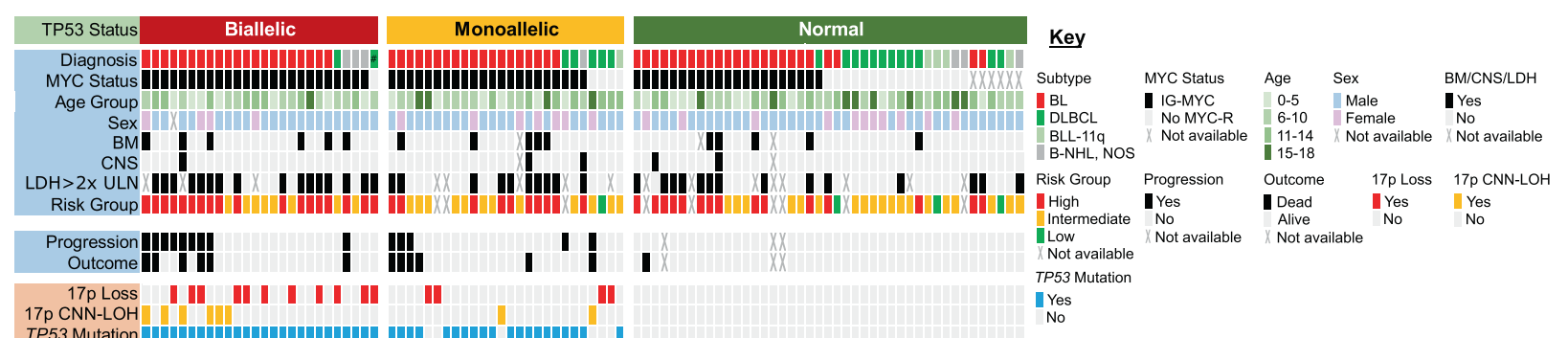

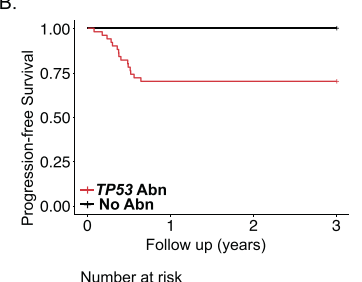

C.

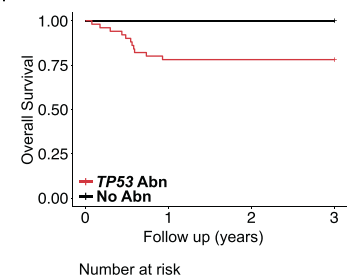

D.

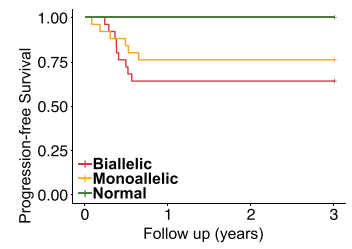

E.

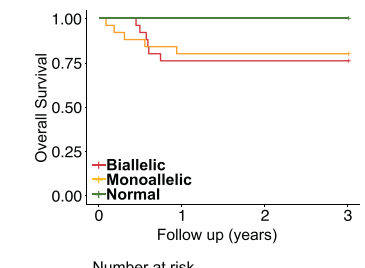

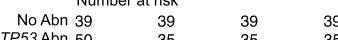
\begin{tabular}{cccc}
\multicolumn{5}{c}{ Number at risk } \\
No Abn 39 & 39 & 39 & 39 \\
TP53 Abn 50 & 39 & 39 & 39
\end{tabular} \begin{tabular}{cccc}
\multicolumn{5}{c}{ Number at risk } \\
Biallelic 25 & 16 & 16 & 16 \\
Monoallelic 25 25 & 19 & 19 & 19 \\
Normal 39 & 39 & 39 & 39
\end{tabular} \begin{tabular}{cccc}
\multicolumn{5}{c}{ Number at risk } \\
Biallelic 25 & 19 & 19 & 19 \\
Monoallelic 25 25 & 20 & 20 & 20 \\
Normal 39 & 39 & 39 & 39
\end{tabular}

Fig. 3 Identification of patient risk groups based on TP53 status. (A) Oncoplot showing TP53 status with clinical and molecular parameters as described in the key. Data is plotted from left to right according to TP53 status. Kaplan-Meier plots showing (B) progression-free and (C) overall survival for any TP53 abnormality (deletion, CNN-LOH and/or mutation), and (D) progression-free and (E) overall survival according to normal (green), monoallelic (amber) or biallelic (red) TP53 status. \# Li-Fraumeni syndrome case.

and may be associated with a greater number of infections [5]. Given the inferior outcome of these patients prior to the use of rituximab and the unacceptable deterioration in that outcome with a previous attempt to reduce therapy intensity [9], they represent a key group for further biomarker-driven stratification.
Therefore, we sought to understand the prognostic impact of TP53 abnormalities specifically in this group. High-risk patients had a higher rate of TP53 abnormality (27/41, 66\%) than low/intermediate-risk groups $(20 / 42,48 \%)$, but here this association was specifically within the TP53 biallelic group $(p=0.037)$ 
A.

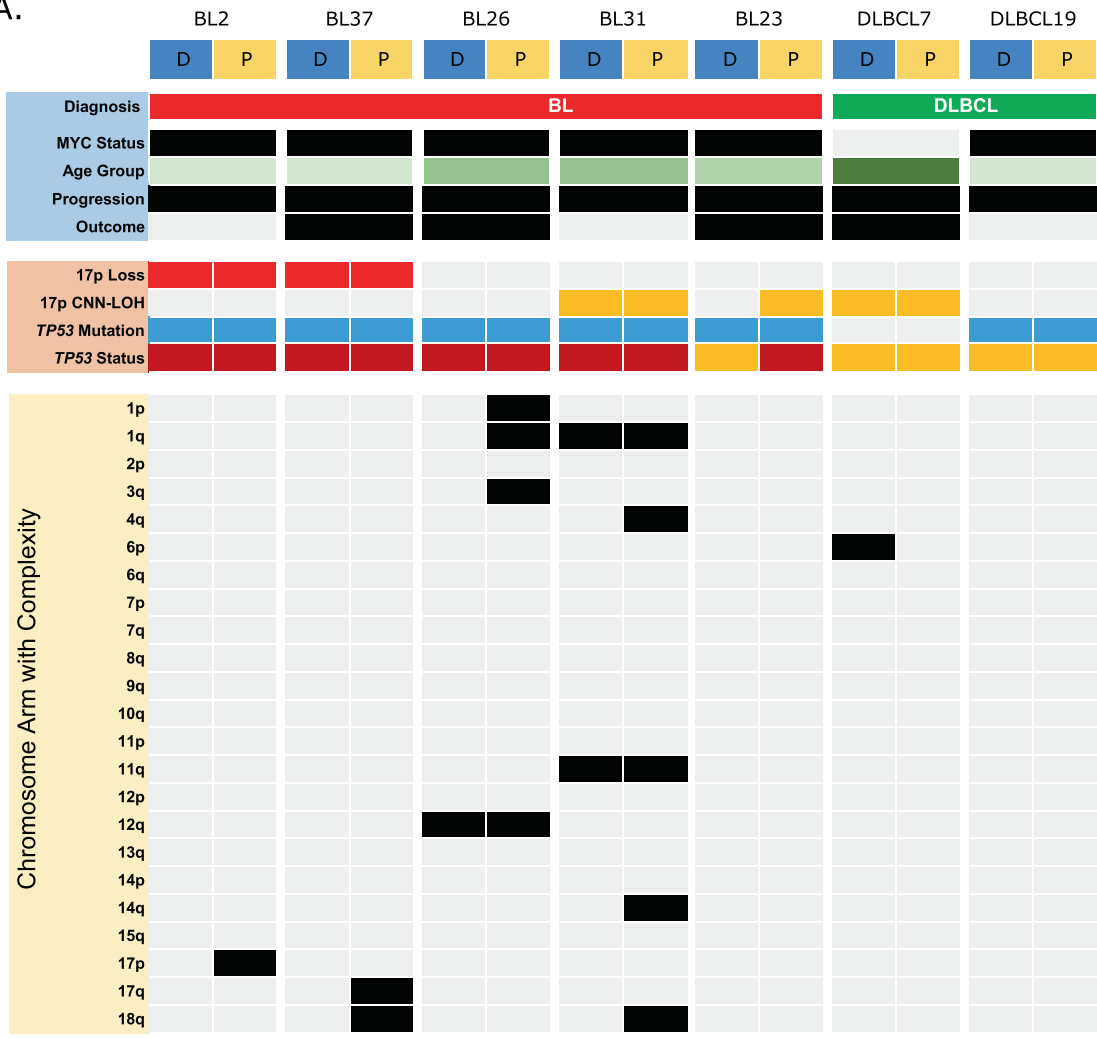

B.

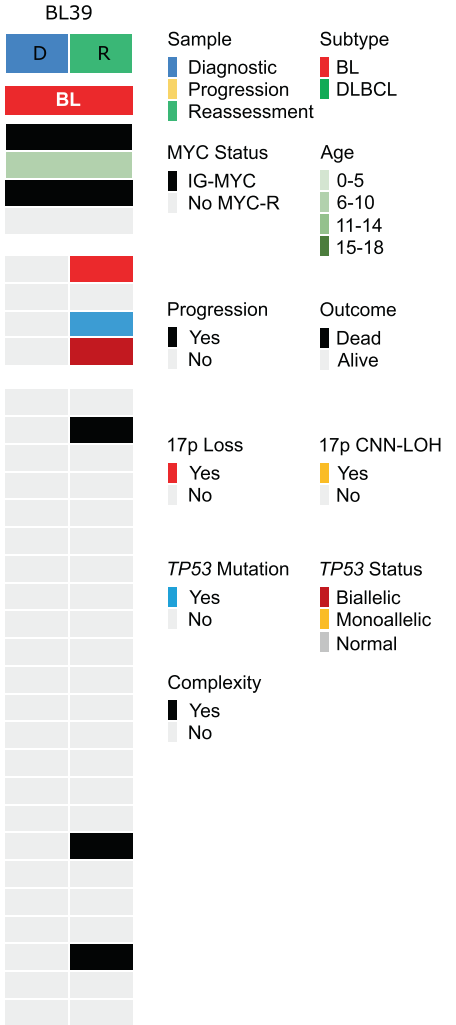

Fig. 5 Biallelic TP53 abnormalities are either maintained or acquired at the time of progression of BL. (A) Overview of clinical and molecular parameters for seven patients with matched samples taken at initial diagnosis (D) and at the time of progression (P). (B) One patient had matched samples from initial diagnosis and the time of reassessment.

unacceptable reduction in survival seen in high-risk bone marrow/ central nervous system positive patients randomised to reduced intensity therapy within the FAB/LMB96 study [9]. Nevertheless, within the reduced intensity arm of the high-risk FAB/LMB96 study, an $80 \%$ EFS was seen, implying that only a minority of patients benefit from intensification of treatment. The inability to identify those with a low risk of treatment failure has hampered risk stratification beyond clinicopathological features. Here, we show that within the high-risk patient group, (as defined in the Inter-B$\mathrm{NHL}$ trial), TP53 wild-type patients have a very low risk of relapse, despite the absence of rituximab therapy. If validated in international trial cohorts, analysis of TP53 status may allow identification of a subset of patients currently considered high-risk for whom chemo-immunotherapy can be de-intensified without compromising efficacy. Initially, this could involve omitting rituximab but further reduction in chemotherapy similar to that attempted within FAB/LMB96, could also be considered. Our finding provides a potential biomarker platform for future trials of therapy reduction, albeit that a large number of patients and a more effective salvage strategy would be necessary for such a trial.

Critical to the better treatment of progressive or relapsed disease is a much deeper understanding of the drivers of therapy resistance. The finding that TP53 abnormalities are associated with increased risk of disease progression, and our analysis of paired Burkitt lymphoma samples showing that all cases either maintained or developed biallelic TP53 abnormalities at progression, suggest a key role for TP53 loss of function in this process. In support of this assertion, Reutter et al. [49] found multiple TP53 abnormalities at diagnosis and relapse in each of the five relapsed
BL cases studied. Given the many biological pathways impacted by $\mathrm{p} 53$, the mechanisms by which TP53 abnormalities promote therapy resistance merits further investigation in support of developing an effective salvage therapy. It is notable, therefore, that we found a strong association between TP53 abnormalities and MYC rearrangements in our cohort. Since these two cancer genes cooperate to drive experimental lymphomagenesis in mice and have been associated with a particularly poor prognosis when concurrently mutated and translocated, respectively, in adult $\mathrm{DLBCL}$, a deeper mechanistic understanding of the interactions between $\mathrm{p} 53$ and MYC in paediatric B-NHL may be particularly informative in this regard $[29,32,38]$.

Mutations, present in half of cases, were the most frequent TP53 abnormalities detected in our cohort. As expected, TP53 deletions and CNN-LOH were less prevalent and were mostly present in tumours with TP53 mutation $[2,50-53]$. Overall, half of all cases with a TP53 abnormality had biallelic alterations expected to abrogate wild-type TP53 functions at diagnosis and biallelic events were present in all BL tumours at the time of progression. However, while the presence of any TP53 abnormality was associated with an increased risk of disease progression and death, there was no difference in PFS or OS between cases with monoallelic or biallelic alterations at diagnosis. This contrasts with recent findings in myelodysplastic syndrome and plasma cell myeloma, in which specifically biallelic TP53 alterations at diagnosis are associated with poor survival [54-56]. The increasing evidence that many mutant p53 proteins exhibit gain of function or dominant-negative properties may provide a partial explanation but our analysis of paired diagnosis/progression BL samples 
suggests the alternative explanation that clones/subclones with monoallelic TP53 abnormalities may evolve to a biallelic state during disease progression under the selective pressure of therapy $[32,33,57]$. Interesting in this regard is the recent report of a case of relapsed $\mathrm{BL}$ with the expansion of a low level (2\% VAF) TP53 $\mathrm{R} 248 \mathrm{Q}$ mutation at diagnosis to a $93 \% \mathrm{VAF}$ at relapse, secondary to a combination of clonal expansion and CNN-LOH [58].

TP53 mutations are associated with several types of genomic instability, including aneuploidy and chromothripsis, and thus increased genome complexity across the cancer spectrum $[33,44,46]$. Notably, a recently described subset of adult DLBCL carrying frequent biallelic inactivation of TP53 by mutation and $17 p$ deletion is selectively associated with an increase in small and large CNAs [21, 45]. Similarly, an association between TP53 abnormalities and chromothripsis-like changes, including of $13 q$, has been reported in adult DLBCL $[59,60]$. Although we did not see an effect on global genomic complexity in our predominantly BL paediatric cohort, we did observe the correlation of TP53 abnormalities with complex copy number patterns involving 1q, $11 q$ and $13 q$. The $13 q$ gains centred on the MIR17HG gene, in keeping with a previous report of an association between $17 p$ deletion and MIR17HG gain in a small sample set [50]. That report speculated that MIR17HG gain was associated with relapse but our data suggest that this probably results indirectly from the association of MIR17HG gain with TP53 abnormalities [50].

We have demonstrated the clinical importance of TP53 abnormalities in paediatric B-NHL, identifying them as a potential biomarker capable of further risk-stratifying patients currently considered high-risk. These findings now need to be validated in a large international trial cohort. For those children without a TP53 abnormality, the risk of disease progression is extremely low and stratified trials examining therapy reduction should be considered. Evolution of biallelic abnormalities at relapse implicates TP53 biology as a driver of therapy resistance and relapse and further understanding of the underlying mechanisms could lead to new strategies to prevent or treat therapy-resistant disease.

\section{REFERENCES}

1. Patte C, Auperin A, Gerrard M, Michon J, Pinkerton R, Sposto R, et al. Results of the randomized international $\mathrm{FAB} / \mathrm{LMB} 96$ trial for intermediate risk B-cell nonHodgkin lymphoma in children and adolescents: it is possible to reduce treatment for the early responding patients. Blood. 2007;109:2773-80.

2. Poirel HA, Cairo MS, Heerema NA, Swansbury J, Auperin A, Launay E, et al. Specific cytogenetic abnormalities are associated with a significantly inferior outcome in children and adolescents with mature B-cell non-Hodgkin's lymphoma: results of the FAB/LMB 96 international study. Leukemia. 2009;23:323-31.

3. Minard-Colin V, Brugieres L, Reiter A, Cairo MS, Gross TG, Woessmann W, et al. Non-Hodgkin lymphoma in children and adolescents: progress through effective collaboration, current knowledge, and challenges ahead. J Clin Oncol: Off J Am Soc Clin Oncol. 2015;33:2963-74.

4. Cairo MS, Sposto R, Gerrard M, Auperin A, Goldman SC, Harrison L, et al. Advanced stage, increased lactate dehydrogenase, and primary site, but not adolescent age $(>/=15$ years), are associated with an increased risk of treatment failure in children and adolescents with mature B-cell non-Hodgkin's lymphoma: results of the FAB LMB 96 study. J Clin Oncol: Off J Am Soc Clin Oncol. 2012;30:387-93.

5. Minard-Colin V, Auperin A, Pillon M, Burke GAA, Barkauskas DA, Wheatley K, et al. Rituximab for high-risk, mature b-cell non-hodgkin's lymphoma in children. $\mathrm{N}$ Engl J Med. 2020;382:2207-19.

6. Ehrhardt MJ, Chen Y, Sandlund JT, Bluhm EC, Hayashi RJ, Becktell K, et al. Late health outcomes after contemporary lymphome Malin de Burkitt therapy for mature B-Cell Non-Hodgkin lymphoma: a report from the childhood cancer survivor study. J Clin Oncol: Off J Am Soc Clin Oncol. 2019;37:2556-70.

7. Attarbaschi A, Carraro E, Ronceray L, Andres M, Barzilai-Birenboim S, Bomken S, et al. Second malignant neoplasms after treatment of non-Hodgkin's lymphomaa retrospective multinational study of 189 children and adolescents. Leukemia. 2020;35:534-49.

8. Srinivasan S, Roy Moulik N, Kc A, Narula G, Sankaran H, Prasad M, et al. Increased toxicities in children with Burkitt lymphoma treated with rituximab: experience from a tertiary cancer center in India. Pediatr Blood Cancer. 2020;67(11):e28682.
9. Cairo MS, Gerrard M, Sposto R, Auperin A, Pinkerton CR, Michon J, et al. Results of a randomized international study of high-risk central nervous system $B$ nonHodgkin lymphoma and B acute lymphoblastic leukemia in children and adolescents. Blood. 2007;109:2736-43.

10. Anoop P, Sankpal S, Stiller C, Tewari S, Lancaster DL, Khabra K, et al. Outcome of childhood relapsed or refractory mature B-cell non-Hodgkin lymphoma and acute lymphoblastic leukemia. Leuk lymphoma. 2012;53:1882-8.

11. Jourdain A, Auperin A, Minard-Colin V, Aladjidi N, Zsiros J, Coze C, et al. Outcome of and prognostic factors for relapse in children and adolescents with mature B-cell lymphoma and leukemia treated in three consecutive prospective "Lymphomes Malins B" protocols. A Societe Francaise des Cancers de I'Enfant study. Haematologica. 2015;100:810-7.

12. Rigaud C, Auperin A, Jourdain A, Haouy S, Couec ML, Aladjidi N, et al. Outcome of relapse in children and adolescents with B-cell non-Hodgkin lymphoma and mature acute leukemia: A report from the French LMB study. Pediatr Blood Cancer. 2019;66:e27873.

13. Woessmann W, Zimmermann M, Meinhardt A, Muller S, Hauch $\mathrm{H}$, Knorr $F$, et al. Progressive or relapsed Burkitt lymphoma or leukemia in children and adolescents after BFM-type first-line therapy. Blood. 2020;135:1124-32.

14. Burke GAA, Beishuizen A, Bhojwani D, Burkhardt B, Minard-Colin V, Norris RE, et al. Ibrutinib plus CIT for R/R mature B-NHL in children (SPARKLE trial): initial safety, pharmacokinetics, and efficacy. Leukemia. 2020;34:2271-5.

15. Pearson ADJ, Scobie N, Norga K, Ligas F, Chiodin D, Burke A, et al. ACCELERATE and European Medicine Agency Paediatric Strategy Forum for medicinal product development for mature B-cell malignancies in children. Eur J Cancer. 2019;110:74-85.

16. Love C, Sun Z, Jima D, Li G, Zhang J, Miles R, et al. The genetic landscape of mutations in Burkitt lymphoma. Nat Genet. 2012;44:1321-5.

17. Schmitz R, Young RM, Ceribelli M, Jhavar S, Xiao W, Zhang $M$, et al. Burkitt lymphoma pathogenesis and therapeutic targets from structural and functional genomics. Nature. 2012;490:116-20.

18. Panea Rl, Love CL, Shingleton JR, Reddy A, Bailey JA, Moormann AM, et al. The whole genome landscape of Burkitt lymphoma subtypes. Blood. 2019;134:1598-607.

19. Grande BM, Gerhard DS, Jiang A, Griner NB, Abramson JS, Alexander TB, et al. Genome-wide discovery of somatic coding and non-coding mutations in pediatric endemic and sporadic Burkitt lymphoma. Blood. 2019;133:1313-24.

20. Lopez C, Kleinheinz K, Aukema SM, Rohde M, Bernhart SH, Hubschmann D, et al. Genomic and transcriptomic changes complement each other in the pathogenesis of sporadic Burkitt lymphoma. Nat Commun. 2019;10:1459.

21. Chapuy B, Stewart C, Dunford AJ, Kim J, Kamburov A, Redd RA, et al. Molecular subtypes of diffuse large $B$ cell lymphoma are associated with distinct pathogenic mechanisms and outcomes. Nat Med. 2018;24:679-90.

22. Schmitz R, Wright GW, Huang DW, Johnson CA, Phelan JD, Wang JQ, et al. Genetics and pathogenesis of diffuse large B-Cell lymphoma. N Engl J Med. 2018;378:1396-407.

23. Reddy A, Zhang J, Davis NS, Moffitt AB, Love CL, Waldrop A, et al. Genetic and functional drivers of diffuse large $B$ cell lymphoma. Cell. 2017;171:481-94.

24. Lacy SE, Barrans SL, Beer PA, Painter D, Smith AG, Roman E, et al. Targeted sequencing in DLBCL, molecular subtypes, and outcomes: a Haematological Malignancy Research Network report. Blood. 2020;135:1759-71.

25. Lohr JG, Stojanov P, Lawrence MS, Auclair D, Chapuy B, Sougnez C, et al. Discovery and prioritization of somatic mutations in diffuse large B-cell lymphoma (DLBCL) by whole-exome sequencing. Proc Natl Acad Sci USA. 2012;109:3879-84.

26. Zhang J, Grubor V, Love CL, Banerjee A, Richards KL, Mieczkowski PA, et al. Genetic heterogeneity of diffuse large B-cell lymphoma. Proc Natl Acad Sci USA. 2013;110:1398-403.

27. Morin RD, Mungall K, Pleasance E, Mungall AJ, Goya R, Huff RD, et al. Mutational and structural analysis of diffuse large B-cell lymphoma using whole-genome sequencing. Blood. 2013;122:1256-65.

28. Pasqualucci L, Trifonov V, Fabbri G, Ma J, Rossi D, Chiarenza A, et al. Analysis of the coding genome of diffuse large B-cell lymphoma. Nat Genet. 2011;43:830-7.

29. Eischen CM, Weber JD, Roussel MF, Sherr CJ, Cleveland JL. Disruption of the ARFMdm2-p53 tumor suppressor pathway in Myc-induced lymphomagenesis. Genes Dev. 1999;13:2658-69.

30. Rowh MA, DeMicco A, Horowitz JE, Yin B, Yang-lott KS, Fusello AM, et al. Tp53 deletion in $B$ lineage cells predisposes mice to lymphomas with oncogenic translocations. Oncogene. 2011;30:4757-64.

31. Xu-Monette $Z Y$, Medeiros $L$, Li Y, Orlowski RZ, Andreeff $M$, Bueso-Ramos $C E$, et al. Dysfunction of the TP53 tumor suppressor gene in lymphoid malignancies. Blood. 2012;119:3668-83.

32. Aubrey BJ, Janic A, Chen Y, Chang C, Lieschke EC, Diepstraten ST, et al. Mutant TRP53 exerts a target gene-selective dominant-negative effect to drive tumor development. Genes Dev. 2018;32:1420-9.

33. Kastenhuber ER, Lowe SW. Putting p53 in Context. Cell. 2017;170:1062-78. 
34. Xu-Monette ZY, Wu L, Visco C, Tai YC, Tzankov A, Liu WM, et al. Mutational profile and prognostic significance of TP53 in diffuse large B-cell lymphoma patients treated with R-CHOP: report from an International DLBCL Rituximab-CHOP Consortium Program Study. Blood. 2012;120:3986-96.

35. Zenz T, Kreuz M, Fuge M, Klapper W, Horn H, Staiger AM, et al. TP53 mutation and survival in aggressive B cell lymphoma. Int J Cancer. 2017;141:1381-8.

36. Intlekofer AM, Joffe E, Batlevi CL, Hilden $\mathrm{P}, \mathrm{He}$ J, Seshan VE, et al. Integrated DNA/ RNA targeted genomic profiling of diffuse large B-cell lymphoma using a clinical assay. Blood Cancer J. 2018;8:60.

37. Xu P, Liu X, Ouyang J, Chen B. TP53 mutation predicts the poor prognosis of nonHodgkin lymphomas: Evidence from a meta-analysis. PLoS One. 2017;12:e0174809.

38. Clipson A, Barrans S, Zeng N, Crouch S, Grigoropoulos NF, Liu H, et al. The prognosis of MYC translocation positive diffuse large B-cell lymphoma depends on the second hit. J Pathol Clin Res. 2015;1:125-33.

39. Campo E, Cymbalista F, Ghia P, Jager U, Pospisilova S, Rosenquist R, et al. TP53 aberrations in chronic lymphocytic leukemia: an overview of the clinical implications of improved diagnostics. Haematologica. 2018;103:1956-68.

40. Swerdlow SH, Campo E, Harris NL, Jaffe ES, Pileri SA, Stein H, et al. WHO Classification of Tumours of Haematopoietic and Lymphoid Tissues, Revised 4th Edition edn. International Agency for Research on Cancer (IARC), 2017.

41. Woessmann W, Seidemann K, Mann G, Zimmermann M, Burkhardt B, Oschlies I, et al. The impact of the methotrexate administration schedule and dose in the treatment of children and adolescents with B-cell neoplasms: a report of the BFM Group Study NHL-BFM95. Blood. 2005;105:948-58.

42. Bouaoun L, Sonkin D, Ardin M, Hollstein M, Byrnes G, Zavadil J, et al. TP53 Variations in Human Cancers: New Lessons from the IARC TP53 Database and Genomics Data. Hum Mutat. 2016;37:865-76.

43. Leroy B, Anderson M, Soussi T. TP53 mutations in human cancer: database reassessment and prospects for the next decade. Hum Mutat. 2014;35:672-88.

44. Donehower LA, Soussi T, Korkut A, Liu Y, Schultz A, Cardenas M, et al. Integrated Analysis of TP53 Gene and Pathway Alterations in The Cancer Genome Atlas. Cell Rep. 2019;28:1370-84.

45. Monti S, Chapuy B, Takeyama K, Rodig SJ, Hao Y, Yeda KT, et al. Integrative analysis reveals an outcome-associated and targetable pattern of $\mathrm{p} 53$ and cell cycle deregulation in diffuse large B cell lymphoma. Cancer Cell. 2012;22:359-72.

46. Eischen CM. Genome Stability Requires p53. Cold Spring Harb Perspect Med. 2016;6:a026096.

47. Salaverria I, Martin-Guerrero I, Wagener R, Kreuz M, Kohler CW, Richter J, et al. A recurrent $11 \mathrm{q}$ aberration pattern characterizes a subset of MYC-negative highgrade B-cell lymphomas resembling Burkitt lymphoma. Blood. 2014;123:1187-98.

48. Aukema SM, Theil L, Rohde M, Bauer B, Bradtke J, Burkhardt B, et al. Sequential karyotyping in Burkitt lymphoma reveals a linear clonal evolution with increase in karyotype complexity and a high frequency of recurrent secondary aberrations. Br J Haematol. 2015;170:814-25.

49. Reutter K, Sandmann S, Rohde J, Muller S, Woste M, Khanam T, et al. Reconstructing clonal evolution in relapsed and non-relapsed Burkitt lymphoma. Leukemia. 2020;35:639-43.

50. Schiffman JD, Lorimer PD, Rodic V, Jahromi MS, Downie JM, Bayerl MG, et al. Genome wide copy number analysis of paediatric Burkitt lymphoma using formalin-fixed tissues reveals a subset with gain of chromosome $13 q$ and corresponding miRNA over expression. Br J Haematol. 2011;155:477-86.

51. Scholtysik R, Kreuz M, Klapper W, Burkhardt B, Feller AC, Hummel M, et al Detection of genomic aberrations in molecularly defined Burkitt's lymphoma by array-based, high resolution, single nucleotide polymorphism analysis. Haematologica. 2010;95:2047-55.

52. Havelange V, Pepermans X, Ameye G, Theate I, Callet-Bauchu E, Barin C, et al. Genetic differences between paediatric and adult Burkitt lymphomas. Br J Haematol. 2016;173:137-44.

53. Scholtysik R, Kreuz M, Hummel M, Rosolowski M, Szczepanowski M, Klapper W, et al. Characterization of genomic imbalances in diffuse large B-cell lymphoma by detailed SNP-chip analysis. Int J Cancer. 2015;136:1033-42.

54. Thanendrarajan S, Tian E, Qu P, Mathur P, Schinke C, van Rhee F, et al. The level of deletion 17p and bi-allelic inactivation of TP53 has a significant impact on clinical outcome in multiple myeloma. Haematologica. 2017;102:e364-e367.

55. Walker BA, Mavrommatis K, Wardell CP, Ashby TC, Bauer M, Davies F, et al. A highrisk, Double-Hit, group of newly diagnosed myeloma identified by genomic analysis. Leukemia. 2019;33:159-70.

56. Bernard E, Nannya Y, Hasserjian RP, Devlin SM, Tuechler H, Medina-Martinez JS, et al. Implications of TP53 allelic state for genome stability, clinical presentation and outcomes in myelodysplastic syndromes. Nat Med. 2020;26:1549-56.

57. Boettcher S, Miller PG, Sharma R, McConkey M, Leventhal M, Krivtsov AV, et al. A dominant-negative effect drives selection of TP53 missense mutations in myeloid malignancies. Science. 2019;365:599-604.
58. Wever CM, Geoffrion D, Grande BM, Yu S, Alcaide M, Lemaire M, et al. The genomic landscape of two Burkitt lymphoma cases and derived cell lines: comparison between primary and relapse samples. Leuk lymphoma. 2018;59:2159-74.

59. Karube K, Enjuanes A, Dlouhy I, Jares P, Martin-Garcia D, Nadeu F, et al. Integrating genomic alterations in diffuse large B-cell lymphoma identifies new relevant pathways and potential therapeutic targets. Leukemia. 2018;32:675-84.

60. Rausch T, Jones DT, Zapatka M, Stutz AM, Zichner T, Weischenfeldt J, et al. Genome sequencing of pediatric medulloblastoma links catastrophic DNA rearrangements with TP53 mutations. Cell. 2012;148:59-71.

\section{ACKNOWLEDGEMENTS}

This study was supported by a Blood Cancer UK Senior Bennett Fellowship (\#12005), the Newcastle upon Tyne Hospitals NHS Charity, North East Promenaders Against Cancer (NEPAC), the JGW Patterson Foundation, the Little Princess Trust, the North of England Children's Cancer Research Fund (NECCR), the Good Will Cause, the Hannah Bloom Charitable Trust, Cancer Research North East, the National Institute for Health Research (NIHR) Newcastle In Vitro Diagnostics Co-operative, the MRC/EPSRC Newcastle Molecular Pathology Node (NovoPath) and MRC Clinician Scientist Fellowship (MR/S021590/1). We thank all the patients, clinicians and staff who participated in the collection of material and clinical data at the Children's Cancer and Leukaemia Group (CCLG) Tissue Bank. The CCLG Tissue Bank is funded by Cancer Research UK

\section{AUTHOR CONTRIBUTIONS}

VR conceived the study, secured funding and finalised the manuscript with editorial assistance from all authors. VR, SB and CMB designed the study, directed the research and wrote the manuscript; $\mathrm{VR}, \mathrm{AMN}, \mathrm{SB}$ and $\mathrm{CMB}$ co-ordinated and participated in data collection, analysis and interpretation; VR, MZ, AEB and AMN processed and analysed the copy number and whole-exome sequencing data; $P Z, A E, A B, R E C$, and SW did laboratory experiments and analysis. VR, $A M N, M Z$ and $A E$ performed statistical analysis; VR, CMB, SB, AB, ST, MT and CJH gathered samples and patient data, and provided clinical interpretation. $C M B, K W$ and DT conducted the pathological review of the cases. All authors approved the manuscript.

\section{COMPETING INTERESTS}

The authors declare no competing interests.

\section{ADDITIONAL INFORMATION}

Supplementary information The online version contains supplementary material available at https://doi.org/10.1038/s41375-021-01444-6.

Correspondence and requests for materials should be addressed to Vikki Rand.

Reprints and permission information is available at http://www.nature.com/ reprints

Publisher's note Springer Nature remains neutral with regard to jurisdictional claims in published maps and institutional affiliations.

\footnotetext{
Attribution 4.0 International License, which permits use, sharing, adaptation, distribution and reproduction in any medium or format, as long as you give appropriate credit to the original author(s) and the source, provide a link to the Creative Commons license, and indicate if changes were made. The images or other third party material in this article are included in the article's Creative Commons license, unless indicated otherwise in a credit line to the material. If material is not included in the article's Creative Commons license and your intended use is not permitted by statutory regulation or exceeds the permitted use, you will need to obtain permission directly from the copyright holder. To view a copy of this license, visit http://creativecommons. org/licenses/by/4.0/.
}

Open Access This article is licensed under a Creative Common

(c) The Author(s) 2021 